1 Universidade Federal da Paraíba (UFPB), Centro de Ciências da Saúde, Departamento de Ciências Farmacêuticas - João Pessoa (PB), Brasil. suelmadefatima@yahoo. com.br

2 Universidade Federal do Rio de Janeiro (UFRJ), Instituto de Estudos em Saúde Coletiva (lesc) - Rio de Janeiro (RJ), Brasil biaensp@gmail.com

3 Fundação Oswaldo Cruz (Fiocruz), Escola Nacional de Saúde Pública Sergio Arouca (Ensp), Departamento de Política de Medicamentos e Assistência Farmacêutica (NAF) - Rio de Janeiro (RJ), Brasil.

dora@ensp.fiocruz.br

4 Fundação Oswaldo Cruz (Fícruz), Escola Nacional de Saúde Pública Sergio Arouca (Ensp), Departamento de Política de Medicamentos e Assistência Farmacêutica (NAF) - Rio de Janeiro (RJ), Brasil.

vera@ensp.fiocruz.br

\section{O modelo de ambiguidade-conflito como ferramenta de análise dos desafios da Assistência Farmacêutica em João Pessoa (PB)}

The ambiguity-conflict model as tool for the analysis of Pharmaceutical Services challenges in João Pessoa, Paraíba, Brazil

Suelma de Fátima Bruns ${ }^{\mathbf{1}}$, Egléubia Andrade de Oliveira ${ }^{2}$, Maria Auxiliadora Oliveira ${ }^{\mathbf{3}}$, Vera Lucia Luiza ${ }^{4}$

RESUMO O objetivo deste artigo é o de analisar os níveis de ambiguidade e conflito em relação aos objetivos e recursos (meios) necessários à implementação da Assistência Farmacêutica no município de João Pessoa (PB). Os dados foram coletados por meio de entrevistas semiestruturadas e sistematizados por meio de análise de conteúdo. Para análise dos resultados, utilizou-se como ferramenta o modelo de ambiguidade-conflito de Richard Matland. Os resultados indicam baixos níveis de ambiguidade sobre os objetivos da política, porém considerável nível de conflito em relação aos meios. Os achados permitiram detectar características de 'Implementação Administrativa', com atributos da tipologia 'Política'.

PALAVRAS-CHAVE Assistência Farmacêutica; Políticas públicas; Avaliação em saúde.

ABSTRACT This paper aims to analyze the levels of ambiguity and conflict related to objectives and resources (means) required for the implementation of Pharmaceutical Services in the city of João Pessoa-PB, Brazil. Data were collected by means of semi-structured interviews and systematized as for content analysis. Data analysis followed the ambiguity-conflict model of Richard Matland. A low ambiguity level was found regarding pharmaceutical policy objectives, but showing a considerable level of conflict in relation to the means. The findings contained some characteristics of 'administrative implementation' and attributes of 'Politics' type.

KEYWORDS Pharmaceutical Services; Public policies; Health evaluation. 


\section{Introdução}

A Assistência Farmacêutica (AF) é um tema recorrente na agenda de governo no Brasil e no mundo, tendo se tornado cada vez mais relevante nas discussões da área da saúde. Sua importância encontra-se ancorada na essencialidade do medicamento para melhoria da saúde e qualidade de vida da população, na promoção do acesso e do uso racional de medicamentos e na composição dos gastos com saúde, que oneram sobremaneira os orçamentos familiares e governamentais. Assim, trata-se de uma política de forte impacto para a sociedade e que impõe grandes desafios ao governo.

Importantes alcances recentes podem ser exemplificados pelo aumento tanto dos recursos financeiros transferidos pela União para aquisição de medicamentos para Estados, Distrito Federal e Municípios, como dos recursos próprios destes entes federados alocados no financiamento de medicamentos. Vieira e Zucchi (2013), utilizando os dados do Sistema Integrado de Gestão de Aquisições (Siga) Brasil, encontraram aumento de 5,5 para 8,9 bilhões de reais no período 2005 a 2009 dos recursos liquidados pelas três esferas de governo, enquanto Aurea et al. (2011), a partir de dados do Sistema Integrado de Administração de Serviços Gerais (Siasg), mostraram um crescimento de 6,4 para 7,3 bilhões de reais entre 2005 e 2008.

Em que pese o aumento dos gastos públicos com medicamento, estes ainda representam carga financeira importante nas despesas familiares em saúde. Os resultados da conta-satélite de saúde indicam que as famílias financiaram $90 \%$ do consumo final de medicamentos, enquanto o sistema público participou com cerca de $10 \%$ em 2007 (IBGE, 2009).

Segundo a Organização Mundial da Saúde (OMS), 50\% de todos os medicamentos prescritos, dispensados ou vendidos são usados inadequadamente (WHO, 2002).
No Brasil, dados do Sistema Nacional de Informações Tóxico-Farmacológicas (Sintox), apontam que os medicamentos ocupam o primeiro lugar entre os agentes causadores de intoxicações em seres humanos e o segundo lugar nos registros de mortes por intoxicação (BRASIL, 2005).

Estudo de avaliação da AF no Brasil demonstra problemas na gestão, dificuldades de acesso e irracionalidade do uso dos medicamentos, o que se reflete em prejuízo à saúde do cidadão, ao seu bolso e ao erário público (OPAS, 2005).

O município de João Pessoa é a capital da Paraíba, um dos estados da região Nordeste. Segundo dados do IBGE (2010), essa Região apresentou os salários mais baixos do Brasil, 2,2 salários mínimos, correspondentes a $71,6 \%$ do salário médio mensal nacional, e o estado da Paraíba, com 1,9 salários mínimos, posicionado entre os três salários mais baixos do nordeste. Portanto, grande parte da população tem reduzido poder aquisitivo, com consequente reflexo na baixa capacidade de aquisição de medicamentos fora do sistema público de saúde.

Diante desse panorama, identificar as barreiras que se interpõem entre a disponibilização dos meios - recursos humanos, financeiros e estruturais -, alcançar os objetivos para cumprimento da missão social torna-se condição fundamental para superar os desafios na institucionalização da AF.

Nesse sentido, este artigo tem como objetivo analisar os níveis de ambiguidade e conflito em relação aos objetivos e recursos (meios) necessários à obtenção dos efeitos esperados com a implementação das ações da AF no município de João Pessoa-PB.

\section{Referencial teórico e metodológico}

O processo de implementação de uma política é caracterizado por disputas entre 
diferentes pontos de vista e permeado pelo alto grau de incerteza que envolve a tomada de decisão, para além da execução de atividades planejadas no âmbito dos programas, políticas ou intervenções. Dessa maneira, os interesses envolvidos e os efeitos resultantes da intervenção dos atores responsáveis pela execução das rotinas - nem sempre alinhados às proposições iniciais - redundam em situações de conflito que irão requerer estratégias de persuasão e a construção de consensos, mesmo que provisórios (SABATIER; JENKINS-SMITH, 1993; CARVALHO, 2006).

$\mathrm{O}$ modelo de ambiguidade-conflito (ambiguity-conflict model) proposto por Matland (1995) para a análise de implementação tem a intenção de superar contradições entre os modelos de análise top-down (focalizam a implementação como decorrência do processo de decisão) e botton up (privilegiam a multiplicidade de atores e sua interação no processo de implementação). Assim, incorpora em sua análise a visão dos formuladores localizados nas esferas centrais de decisão, bem como o papel dos principais interessados (stakeholders) na política ou programa e seus implementadores. Ainda, permite o cotejamento dessas abordagens voltadas ora à apreensão de aspectos das esferas macro-institucionais que influenciam o desenvolvimento de políticas e programas ora sinalizando a importância de fatores micro-institucionais em seu desenho e implementação (CARVALHO, 2006).

Para Matland (1995), o conceito de ambiguidade - situação que ocorre quando existem muitas alternativas ou possibilidades para pensar as mesmas circunstâncias ou fenômenos - materializa-se através de duas dimensões: a 'ambiguidade nos objetivos' (ambiguity of goals), que pode contribuir para o grau de incertezas, e a 'ambiguidade nos meios' (ambiguity of means), relacionada aos recursos necessários (humanos, financeiros e estruturais), concebidos de forma ampla, a serem utilizados nos processos de implementação. Carvalho (2006), tomando por base o modelo de Matland (1995), desenvolveu quadro explicativo relacionando os conceitos e o tipo de implementação.

Numa apresentação abreviada, os quatro tipos de implementação estão dispostos no quadro 1, quais sejam: (1) Implementação Administrativa - política com baixos níveis de ambiguidade e de conflito, que se caracteriza por centrar o foco nos recursos; tem conhecimento dos objetivos e meios; baixo uso de coerção; ações em ambiente estável; pouca ou nenhuma influência externa; fluxo de orientação ordenado hierarquicamente; falhas vistas como problemas técnicos, inadequação dos recursos, má compreensão, escassez de tempo, coordenação inadequada; (2) Implementação Política - política com baixo nível de ambiguidade e alto nível de conflito, que se caracteriza pela ideia de que 'os resultados da implementação são decididos pelo poder'; disputas sobre os meios de implementação; forte influência do contexto político; uso de coerção; boicote e recusa em participar - pode ocorrer no nível local; implementador nem sempre mantém contato direto com a hierarquia superior; (3) Implementação Experimental - política com alto nível de ambiguidade e baixo nível de conflito; foco nos recursos contextuais; objetivos e meios não são claros ou objetivos claros e meios não; aprendizagem como meta, mas não obrigatoriamente; resultado depende dos atores e de recursos do ambiente da micro implementação; mais aberta a influências locais; há casos em que as preferências são problemáticas e a tecnologia é incerta; e (4) Implementação Simbólica - política com alto nível de ambiguidade e alto nível de conflito; foco na força da coalizão; superação do conflito (coerção ou barganha); ambiguidade dificulta estruturação e monitoramento local; ambiguidade pode levar a resultados diferentes, em locais diferentes; conflito provoca decisão local de natureza política; conflito pode ser positivo, para busca de solução; em geral, pouco efeito, mas muita exposição. 
Quadro 1. Conceitos e tipos de implementação do modelo ambiguidade-conflito

\begin{tabular}{|c|c|c|c|c|c|}
\hline \multirow[t]{2}{*}{ Conceitos } & \multirow[t]{2}{*}{ Dimensões } & \multicolumn{4}{|c|}{ Tipos de Implementação } \\
\hline & & Administrativa & Política & Experimental & Simbólica \\
\hline Ambiguidade & $\begin{array}{l}\text { De objetivos } \\
\text { De meios }\end{array}$ & Baixa & Baixa & Alta & Alta \\
\hline Conflito & $\begin{array}{l}\text { Interdependência entre atores } \\
\text { Incompatibilidade de objetivos / meios }\end{array}$ & Baixo & Alto & Baixo & Alto \\
\hline
\end{tabular}

Fonte: Modelo de Matland (1995) adaptado de Carvalho (2006).

\section{Materiais e método}

Os dados utilizados neste estudo são provenientes de pesquisa mais ampla (BRUNS, 2013), na qual a abordagem aqui apresentada foi triangulada com indicadores obtidos em uma amostra de 32 Unidades de Saúde da Família (USF).

Neste artigo, analisam-se os dados correspondentes às 22 entrevistas, realizadas entre fevereiro e maio de 2012 por meio da aplicação de roteiros semiestruturados. Entrevistaramse quatro gestores da secretaria de saúde do Município, oito farmacêuticos, cinco médicos e cinco entregadores de medicamentos, selecionados de forma intencional e não probabilística. Esse processo de seleção objetivou que a amostra fosse constituída por profissionais de diferentes níveis de atuação diretamente envolvidos com a AF básica do Município, de modo a captar diversas perspectivas, tanto de atores localizados nas esferas centrais de decisão como nas de implementação. Dado que a dispensação de medicamentos é considerada uma atividade privativa do farmacêutico, no Brasil, os demais profissionais que a realizam são designados como 'entregadores de medicamentos'. De maneira a localizar o tipo de fala, porém preservando o compromisso de sigilo, as falas estão identificadas por códigos, da seguinte forma: gestor (G1 ao G4); farmacêutico atuando no cuidado (F1 a F8); médicos ( $\mathrm{M} 1$ a M5); e entregadores de medicamentos (E1 a E5).

$O$ roteiro da entrevista continha questões referentes ao grau/nível de implementação da política de AF; à lógica e à logística do ciclo dessa política; à percepção dos atores quanto ao tipo de AF prestada à população; $\mathrm{e}$ desafios e dificuldades percebidas.

Aplicou-se o método de análise de conteúdo (BARDIN, 2011), que abrangeu as etapas de pré-análise, exploração das transcrições, tratamento e interpretação dos resultados. Utilizou-se a modalidade temática, por meio de categorização, que

consiste em descobrir os 'núcleos de sentido' que compõem a comunicação e cuja presença e frequência de aparição podem significar alguma coisa para o objeto analítico escolhido. (BARDIN, 2011, P. 35).

Definiram-se as seguintes categorias: 1 . Sobre a clareza dos objetivos da AF; 2. Meios para consecução da implementação da $\mathrm{AF}$ municipal (recursos humanos, financeiros e estruturais); 3. Interdependência entre os atores; 4. (In)compatibilidade de objetivos e meios. As variáveis centrais - ambiguidade e conflito, previstas no modelo, nortearam a análise das categorias temáticas, tendo como foco os desafios e as dificuldades relatadas pelos entrevistados para implementação da AF no município de João Pessoa (PB), como podem ser observadas no (quadro 2). A identificação das dimensões e componentes utilizados na análise dos níveis de ambiguidade-conflito presentes na implementação foi adaptada de Carvalho (2006). A organização do texto buscou atender ao protocolo COREQ de pesquisa qualitativa (TONG; SAINSBURY; CRAIG, 2007). 
Quadro 2. Dimensões, componentes e atividades propostas para a análise dos desafios para implementação da Assistência Farmacêutica no município de João Pessoa (PB)

\begin{tabular}{cll}
\hline Conceitos & \multicolumn{1}{c}{ Dimensões } & \multicolumn{1}{c}{ Componentes } \\
\hline Ambiguidade & Metas & Clareza quanto aos objetivos da AF pelos atores \\
& Meios & $\begin{array}{l}\text { Influência de fatores locais (percepção de importância da AF, percepção } \\
\text { de suficiência de recursos humanos, estruturais e financeiros) }\end{array}$ \\
\hline Conflito & Relação entre os atores & Existência de conflitos e mecanismos de superação \\
& Incompatibilidade de objetivos & Não concordância quanto aos objetivos/meios \\
\hline
\end{tabular}

Fonte: Elaboração própria.

Todos os entrevistados assinaram Termo de Consentimento Livre e Esclarecido (TCLE), autorizando a gravação dos depoimentos concedidos. Os entrevistados são referidos por códigos de letras. O projeto foi aprovado pelo Comitê de Ética em Pesquisa da Escola Nacional de Saúde Pública Sergio Arouca sob Protocolo de Pesquisa CEP/Ensp - No 265/11 e CAAE: 0282.0.031.000-11.

\section{Resultados}

A partir dos depoimentos concedidos, observaram-se temas comuns no corpus do texto, o que permitiu elencar as quatro categorias emergentes e possibilitou o reagrupamento das falas em torno dessas categorias, identificando os desafios da AF municipal na compreensão dos entrevistados.

\section{Sobre a clareza dos objetivos da Assistência Farmacêutica}

Nessa categoria, observou-se se os entrevistados tinham clareza quanto aos objetivos primordiais da $\mathrm{AF}$, com destaque à garantia do acesso aos medicamentos pela população e ao seu uso racional.

Os gestores e os farmacêuticos mostraram confluência de opinião.

[...] a gente quer abastecer a todos e racionalizar o uso para que os pacientes tenham um medicamento certo, na hora certa, na dose certa e que ele faça o uso correto, completo, que ele comece e termine o tratamento, isso pra gente é muito importante (G1).

"Ações para desenvolver o uso racional de medicamentos [...] material de orientação $e$ educação continuada direcionada aos usuários ou profissionais, não tem" (F4).

Outros profissionais entrevistados, embora compreendessem os objetivos finalísticos da AF relacionados ao acesso a medicamentos, não demonstraram clareza quanto ao uso racional. "Ações para desenvolver o uso racional do medicamento? A gente prefere a quantidade do medicamento não é, [...] eu nunca coloco a mais [...] eu dou o tratamento" (M1). "Eu vejo o papel da AF como política de saúde muito importante para toda a comunidade, [...] principalmente na questão do acesso" (E1).

\section{Meios para consecução da implementação da Assistência Farmacêutica municipal (recursos humanos, financeiros e estruturais)}

Para que uma política pública seja implementada com êxito, é fundamental um adequado planejamento dos recursos (humanos, financeiros e estruturais) necessários. Os entrevistados enfatizaram a necessidade premente do farmacêutico nas farmácias das USF, bem como problemas relacionados a aspectos quantitativos e qualitativos dos recursos humanos da AF. 
É fundamental a presença do farmacêutico na questão do controle na farmácia, na dispensação e na orientação, como também ele estar inserido na equipe para solucionar determinados casos e melhorar os serviços prestados a população. (F8).

"[...] vejo como obstáculo não ter um farmacêutico, [...] a gente precisa do farmacêutico junto, a gente precisa de orientação, o médico não é o dono da verdade" (M2). "[...] a gente tem conflitos pela falta de conhecimento dos profissionais do que venha a ser uma Política de AF, a maioria não conhece como a política acontece [...]" (G2).

Os entrevistados relataram que há disponibilidade de recursos financeiros para a aquisição dos medicamentos padronizados pelo Município. Entretanto, demonstraram preocupação com o orçamento previsto e destinado a essas aquisições, em virtude da crescente demanda judicial a ser atendida. Foi consenso entre eles a necessidade de investimentos na infraestrutura e contratação de farmacêuticos para as USF. "O Financeiro para medicamentos eu acho satisfatório” (M4).

[...] muitas vezes nós temos que fazer complementação com os recursos próprios para a aquisição de insumos e de materiais relativos à $A F$, inclusive para adquirirmos medicamentos não padronizados e para atender às ações judiciais, que, por vezes, desestruturam completamente o nosso planejamento orçamentário, teto financeiro. (G2).

“[...] são necessários mais investimentos para contratação de farmacêuticos e estruturação dos serviços [...]" (F3).

Quanto aos recursos estruturais, os entrevistados relataram inadequações na grande maioria das farmácias das USF e referem como principais problemas o espaço físico insuficiente e inadequado; a falta de refrigeração; e infiltrações, que influenciam na conservação e qualidade dos medicamentos. Contudo, elogiaram as novas USF integradas, recém-construídas.

[...] têm unidades que ainda funcionam em prédios alugados numa situação horrível, com muita infiltração, mal estruturada, a farmácia dividindo

espaço com outras coisas. (F8).

Onde está o grande nó é nos serviços de farmácia, [...] que não têm uma estrutura adequada; é totalmente precária [...] e isso desestimula, porque não tem um local ideal, influencia no processo de trabalho [...]. Agora nas unidades integradas são outra história, já melhora muito. (F7).

\section{Interdependência entre os atores}

$\mathrm{Na}$ opinião dos entrevistados, as principais barreiras ao desenvolvimento da AF envolvem o estabelecimento de consenso entre gestores e implementadores acerca das prioridades a serem adotadas para a melhoria dos serviços, bem como a integração dos atores e ações envolvidas para implementação de tal política.

O conflito é a gente chegar num consenso de prioridade, [...] conseguirmos falar a mesma linguagem, interagir um pouco mais, principalmente com os prescritores, [...] e o povo precisa dessa interação entre os profissionais de saúde para que haja resolutividade na atenção prestada. (G1).

[...] precisa sensibilizar os atores, não adianta ficarem só os farmacêuticos discutindo, precisa da integração do médico, do enfermeiro, do funcionário, da gestão, do usuário, para estarmos juntos, entendendo um pouco mais da Política. (G3).

O nosso conflito é fazer o usuário entender a prescrição: muitas vezes passamos uma medicação para sete dias e no 4ำ dia o cidadão para de tomar. A superação disso ai só vai acontecer com a mão de obra especializada (farmacêutico), com todos trabalhando juntos. (M3).

Os entrevistados problematizaram a ideia de que uma abordagem interdisciplinar 
potencializa novas propostas de ação e intervenção, bem como amplia o poder de articulação com as esferas decisórias, concorrendo para a melhoria dos programas.

Dificuldades para articular as ações, nós temos desde o processo de trabalho da equipe de saúde da família, [...] há muitos profissionais que não se comprometem e acabam fazendo de acordo com seu próprio interesse e isso é muito frágil para o processo político. Isso seria a micro política, e é nisso que nós temos que investir. (G2).

Em virtude da complexidade que envolve a AF, é fundamental que o planejamento e a execução de todas as etapas que compõem o ciclo da AF (Seleção, Programação, Aquisição, Armazenamento, Distribuição, Controle de Estoque, Dispensação e Orientação Farmacêutica) sejam realizadas de forma sistemática e integrada. Sobre esses aspectos do ciclo da AF, os entrevistados reforçam importantes desafios.

A AF é uma área ainda muito fragmentada; $a$ gente precisa ter o farmacêutico presente em todas as unidades de saúde e em todas as etapas do ciclo da $A F$, o que hoje em dia ainda não acontece $[\ldots]$. (F3).

[...] até hoje, nós não temos uma Remume [Relação Municipal de Medicamentos] e nem uma CFT [Comissão de Farmácia e Terapêutica] instituída no município; isso ainda é um gargalo muito grande. (G2).

"[...] uma CFT para realmente discutir essa seleção de medicamentos [...]” (G3).

Relataram-se dificuldades para a realização de uma programação de medicamentos confiável para o Município em virtude da falta de uma série histórica do controle de estoque e do desabastecimento da rede.

Eu acho importante a questão do controle do estoque, mas hoje ainda é uma coisa muito deficitária, [...] sem controle de estoque a gente não consegue fazer um planejamento, uma programação, não se consegue fazer nada disso e aí as faltas acontecem. (G1).

"[...] muitos agentes administrativos têm dificuldade de construir um mapa de medicamentos; eu não sei ao certo o consumo daquela unidade, sempre fica faltando ou fica sobrando" (F8).

Quanto à aquisição de medicamentos, os entrevistados foram unânimes em afirmar que a burocracia envolvida nos processos licitatórios traz muitas dificuldades, principalmente pela morosidade dos processos, problemas com a eleição de prioridades e com o cumprimento dos prazos. $\mathrm{O}$ atendimento a demandas de medicamentos não padronizados e o grande volume de mandados judiciais, o que mobiliza tempo dos profissionais num contingente já extremamente reduzido, foi ressaltado como um foco de desgaste permanente.

[...] os processos licitatórios são extremamente lentos [...] um pregão para poder acontecer tem que ser fechado em três meses [...] mas não, nós não temos prazos, não temos limites, passa um ano ou mais [...], e ai foge completamente de nossa governabilidade. (G1).

O entrave maior são as licitações, porque, às vezes, o recurso tem, mas por alguma ordem jurídica, administrativa ou burocrática acaba emperrando e o medicamento não chega ao usuário. (F7).

Outro ponto ressaltado pelos entrevistados foram os problemas quanto à dispensação e orientação farmacêutica. Eles se mostraram preocupados tanto com a entrega dos medicamentos feita por pessoas não habilitadas como com a falta de orientação farmacêutica aos pacientes. Não ocorre dispensação; o que ocorre é a entrega de medicamentos sem orientação. "Os farmacêuticos que deviam estar presentes ainda não estão [...]” (F8). "[...] um farmacêutico dando orientação em relação à posologia e às interações medicamentosas, isso necessita, porque não acontece aqui na nossa realidade” (E4). 


\section{(In)compatibilidade de objetivos e meios}

Uma das incompatibilidades entre objetivos e meios, reveladas pelos entrevistados, diz respeito à ampliação do acesso, visto que a maior reclamação dos usuários da saúde é a falta de medicamentos, mesmo que temporária, e a centralização dos medicamentos de controle especial nos Centros de Atenção Integral à Saúde (Cais). Um dos dilemas está relacionado com a presença do profissional farmacêutico nas USF, mandatória quando há medicamentos controlados. "O que a população mais reclama é a falta de medicamentos, [...]” (G3).

A reclamação é a falta de alguns medicamentos e dos medicamentos controlados, porque os idosos precisam pegar em lugares muito longe e a maioria não têm condic̦ões físicas para talou dinheiro para pagar a passagem. (F6).

“[...] ampliar o acesso, trazer a parte de mais emergência (medicamentos injetáveis e controlados) para as USF, então, tem que ter um farmacêutico" (M3).

Alguns relatos afirmam que a política de AF atualmente implementada se encontra em fase de construção. Isto requer, portanto, maior estruturação e fortalecimento para garantir o cumprimento de seus objetivos, necessitando que sejam disponibilizados os meios para que a implementação aconteça de forma satisfatória.

[...] necessidade de se ter um farmacêutico para orientar o paciente no ato da dispensação de medicamentos, porque onde tem medicamento tem que ter farmacêutico, isso é fato, é legislação. Quem está fazendo essa orientação? Não é o farmacêutico. (G1).

Na questão de recursos estruturais das farmácias das USF, infelizmente não temos estruturas adequadas; a farmácia fica meio esquecida lá no fundo perto do banheiro, da cozinha; infelizmente a gente se depara com isso, porque também não tem o olhar da importância que é a parte da farmácia, só serve para estocar mesmo! Eu fico horrorizada quando vejo isso. (F1).

Os entrevistados também fizeram referência à limitação do conhecimento por parte de alguns profissionais da saúde e da população sobre o papel e a importância dessa política para a melhoria da qualidade de vida da população. Igualmente, a existência de profissionais não capacitados desenvolvendo funções importantes na AF é um problema adicional.

[...] é uma área ainda muito fragmentada [...], então, a gente não pode exigir dos profissionais (vigia, servente, auxiliar administrativo) que não são da nossa área e que não foram capacitados para isso. (F3).

[...] eles não conhecem (medicamento) pelo nome genérico, não sabem fazer uma troca, não sabem dar uma informação ao usuário, que, muitas vezes, sai sem o medicamento, tendo o medicamento lá, $[. .$.$] isso complica, fora outros$ agravantes que podem acontecer. (F4).

[...] a população não sabe o que é $A F$, acha que é só pegar e entregar, se tem recebe e vai embora e se não tem reclama, [...] muitos funcionários daqui não sabem, imagine a população que não é esclarecida. (F1).

\section{Discussão}

A AF é definida como um conjunto de ações voltadas à promoção, proteção e recuperação da saúde, tanto no nível individual como coletivo, tendo o medicamento como insumo essencial e visando ao acesso e a seu uso racional (BRASIL, 2004). Nesse contexto, convergimos a discussão em dois eixos estratégicos: os objetivos basilares dessa política (acesso e o uso racional de medicamentos) e os meios (recursos humanos, financeiros e estruturais) necessários para sua implementação. 
Verificou-se baixo nível de ambiguidade com relação aos objetivos da política de AF, visto que os entrevistados demonstraram compreender o papel e importância da AF na qualidade da assistência prestada aos usuários. Entretanto, reconhecem que, apesar dos avanços em termos do acesso da população a medicamentos, o desabastecimento ainda é uma realidade constante no Município e gera insatisfação. A baixa disponibilidade relatada pelos entrevistados foi também detectada por Bruns (2013) em 32 farmácias das USF avaliadas em João Pessoa, cuja disponibilidade média de medicamentos-chave variou de $41,1 \%$ a $94,1 \%$ de fevereiro a abril de 2012 .

Outro objetivo essencial da AF é a promoção do Uso Racional de Medicamentos (URM), que compreende uma prescrição adequada, disponibilidade oportuna, dispensação orientada e em condições apropriadas, consumo nas doses certas, pelo período indicado e nos intervalos definidos de medicamentos eficazes, seguros e de qualidade (SANTOS; NITRINI, 2004). Segundo os entrevistados, até o momento da pesquisa, nenhuma ação nesse sentido havia sido desenvolvida no Município e, embora gestores e farmacêuticos tivessem ciência do seu significado, os prescritores pareciam desconhecê-lo, o que pode dificultar a implementação dessas ações e a cooperação desses atores na sua institucionalização.

No que concerne à prescrição adequada, a posição dos profissionais envolvidos nesse ato permite inferir uma baixa integração entre os atores com responsabilidade na cadeia de implementação da política. Prescritores e farmacêuticos, falando de locais distintos, expressaram as dificuldades de interação entre profissionais cuja atuação hierarquizada dificulta a cooperação entre os saberes específicos que poderiam confluir para a qualidade da $\mathrm{AF}$ prestada. Uma proporção importante de prescritores desconhece os possíveis efeitos nocivos do que prescreve ou mesmo não sabe identificar nem prevenir corretamente combinações perigosas entre as substâncias farmacológicas (NASCIMENTO, 2003). Portanto, a falta de informação segura para prescritores, entregadores e usuários; a propaganda distorcida de medicamentos; a ausência da orientação farmacêutica; e a cultura da polifarmácia e da automedicação concorrem para ampliar os níveis de ambiguidade envolvendo o uso racional de medicamentos.

Desse modo, tanto para a equipe multiprofissional de saúde como para a comunidade, é de grande relevância a disseminação e apreensão desse conceito para minimizar os danos advindos da irracionalidade do uso de medicamentos, realidade considerada um problema de saúde pública em todo o mundo (LE GRAND; HOGERZELL; HAAIJER-RUSKAMP, 1999).

Para Marin et al. (2003) a visão sistêmica do ciclo de AF surge como estratégia para superar a fragmentação da AF no Brasil - concentrada apenas nas ações de aquisição e distribuição -, estabelecendo fluxos na construção de um conjunto integrado, que influencia e é influenciado por todas as suas etapas, sendo estas permeadas por mecanismos de gestão e adotadas como princípio norteador da AF no Sistema Único de Saúde (SUS).

Os entrevistados relataram entraves que dificultam a operacionalização satisfatória do ciclo da AF, gerando conflitos entre gestores e farmacêuticos. Tais conflitos se expressam nas fragilidades verificadas na programação consolidada, controle de estoque, condições inadequadas de conservação dos medicamentos e na logística que envolve os processos licitatórios. Além desses aspectos, a dispensação de medicamentos vem sendo realizada por profissionais não capacitados e existem barreiras de acesso impostas pela concentração da dispensação de alguns medicamentos, como os de controle especial, ofertados apenas nos três Cais. Todas essas situações se apresentaram como foco de tensão permanente entre os profissionais diretamente envolvidos, revelando a disputa com relação aos meios a serem mobilizados para a implementação da AF. 
Tais sinalizações, embora vindas de atores distintos, merecem destaque nos processos de revisão e realinhamento das ações, uma vez que repercutem negativamente nas etapas do ciclo da AF como um todo, com impactos tanto à gestão como nas ações de saúde prestadas à população. Para que a AF seja efetivamente implementada, faz-se premente a disponibilização dos meios necessários para sua institucionalização e alcance dos objetivos. Portanto, a disponibilidade de forma planejada, continuada e adequada dos recursos humanos, financeiros e estruturais são elementos indispensáveis de sucesso, uma vez que, sendo uma decisão política, precisa ser encampada pelo gestor da organização, que deve convergir com seu plano e sua capacidade de governo (MARIN ET AL., 2003).

Os farmacêuticos, considerados $\mathrm{O}$ principal ativo das organizações da $\mathrm{AF}$, encontram-se alocados em funções técnico-administrativas e gerenciais, em detrimento das funções assistenciais. Essa condição colide com a compreensão dos objetivos da AF expressa nos depoimentos, uma vez que a dispensação e orientação farmacêutica não podem prescindir da presença do profissional habilitado para desempenhar essa atividade. Nesses termos, as informações sobre os objetivos da política, embora produzam menos incerteza, não reduzem o nível de ambiguidade de uma decisão. A situação foi demonstrada por Bruns (2013), em outro estudo no mesmo Município, onde o farmacêutico foi encontrado desenvolvendo suas atividades de forma permanente em apenas duas de 32 unidades de saúde visitadas.

Os entregadores de medicamentos foram ouvidos com a intenção de captar a percepção desse profissional sobre a política em questão, uma vez que o envolvimento nas decisões varia conforme as relações estabelecidas entre o indivíduo e a organização onde se encontra. A maioria desses profissionais não apresenta formação específica em saúde nem foi preparada para o desempenho de suas atividades, além de mostrar pouca percepção do seu papel no desenvolvimento das ações. O compromisso dos implementadores com os objetivos da política e sua habilidade em utilizar os recursos disponíveis são fatores críticos no processo de implementação. Urge, na opinião dos entrevistados, o desenvolvimento de um programa de educação continuada para ampliar o aprendizado organizacional e individual sobre políticas e programas sociais, além das questões específicas da AF.

Com relação aos recursos financeiros, os entrevistados defenderam a necessidade de mais investimento em infraestrutura e contratação de farmacêuticos e acreditam que a falta de medicamentos não ocorre por problemas financeiros e sim burocráticos. Contudo, de acordo com o Conass (2011), um problema comum aos gestores do SUS é o subfinanciamento da saúde, com repercussões na área da AF, pois, somente com financiamento assegurado é possível disponibilizar os medicamentos e viabilizar o desenvolvimento e continuidade das ações nesta área.

Ademais, os gestores demonstraram grande preocupação com o número crescente de demandas judiciais, que consome parcela expressiva do orçamento previsto para a aquisição de medicamentos na rede estudada. De fato, a judicialização da AF tem se mostrado um dos motivos que desorganiza e desequilibra os recursos financeiros do sistema de saúde, podendo inviabilizar o planejamento das ações de saúde e comprometer o orçamento para aquisição de medicamentos (BRUNS; LUIZA; OLIVEIRA, 2013).

Quanto aos recursos estruturais, a grande maioria das farmácias das USF foi considerada inadequada, embora as USF recém-construídas tenham sido elogiadas. As instalações não apresentam condições para o armazenamento e dispensação de medicamentos, pois são adaptadas em casas alugadas, com espaço físico e refrigeração insuficientes. As duas realidades diametralmente opostas vivenciadas pelos usuários - USF novas e adequadas vis-à-vis antigas e desestruturadas 
- são um foco de insatisfação e desestímulo para os profissionais de saúde.

Como uma das limitações da pesquisa, sabe-se que a explicação a partir da opinião e percepção de pessoas poderá variar com a mudança de atores e que os mesmos respondentes podem mudar de posição ao longo do tempo. A primeira autora residia no Município estudado e já desempenhou cargo público na Prefeitura, ainda que não na Secretaria de Saúde, o que pode ter influenciado as falas.

\section{Conclusão}

A ambiguidade e o conflito têm papel preponderante, influenciando o processo decisório das organizações. Sob essa perspectiva, incorporando na análise tanto o ponto de vista dos decisores centrais como a percepção dos implementadores e, ainda, considerando o conhecimento sobre os objetivos e meios, podem-se detectar características do tipo 'Implementação Administrativa', que apresentam baixos níveis de ambiguidade e conflito no que concerne aos objetivos, metas e fluxos da política, as falhas vistas como problemas técnicos, cujos resultados estão diretamente vinculados à disponibilidade de recursos.

Adicionalmente, considerando o papel da influência externa na cadeia de implementação e, ainda, o ambiente sujeito a

\section{Referências}

AUREA, A. P. et al. Programas de Assistência Farmacêutica do governo federal: estrutura atual, evolução dos gastos com medicamentos e primeiras evidências de sua eficiência, 2005-2008. Brasília, DF: IPE, 2011.

BARDIN, L. Análise de Conteúdo. Lisboa: Edições 70, 2011.

BRASIL. Ministério da Saúde. Agência Nacional de Vigilância Sanitária. Anvisa faz parceria para diminuir mau uso de medicamentos. 2005. Disponível em: <http://www.anvisa.gov.br/divulga/noticias/2005/181105.htm>. Acesso em: 30 mar. 2015. instabilidades, podem-se registrar atributos de uma tipologia 'Política', onde os resultados da implementação são decididos pela esfera central do Município, sofrendo influências do contexto político e condicionados por um elevado nível de conflito em relação aos recursos, tecnologias e meios para o cumprimento das diretrizes definidas. Nesse caso, além de disputas internas envolvendo opositores da política, os implementadores nem sempre estabelecem contato direto com a hierarquia superior. Os resultados e efeitos obtidos nesse caso dependem tanto de recursos quanto de atores que atuam na esfera da micro implementação em seus diferentes contextos.

Buscando apreender as diversas nuances que perpassam a implementação de uma política tão complexa como a da AF, acredita-se que o estudo tenha proporcionado um olhar diferenciado da implementação, cotejando as contribuições do campo da análise de políticas, particularmente à luz das reflexões de Matland (1995).

Portanto, ficou evidenciado que, apesar dos avanços observados quanto à implementação dessa política, ainda se faz necessário um importante esforço de governantes, gestores e técnicos para o alcance dos objetivos primordiais relacionados ao acesso e uso racional de medicamentos, garantindo melhor qualidade de vida à população.
—. Ministério da Saúde. Resolução n 338, de 6 de maio de 2004. Aprova a Política Nacional de Assistência Farmacêutica. Diário Oficial [da] União. Brasília, DF, 2004. Disponível em: < http:// bvsms.saude.gov.br/bvs/saudelegis/cns/2004/ res0338_06_05_2004.html>. Acesso: 19 out. 2015.

BRUNS, S. F. Política de assistência farmacêutica no município de João Pessoa-PB: contexto, desafios e perspectivas. 2013. 258 f. Tese (Doutorado em Saúde Pública) - Fundação Oswaldo Cruz, Escola Nacional de Saúde Pública Sergio Arouca, Rio de Janeiro, 2013. 
BRUNS, S. F.; LUIZA, V. L.; OLIVEIRA, E. A. Judicialização da assistência farmacêutica: dois pesos e uma medida. In: OLIVEIRA, M. H. et al. (Org.). Direito e Saúde: cidadania e ética na construção de sujeitos sanitários. Maceió: EDUFAL, 2013, p. 366-390.

CARVALHO, E. H. Programa de Saúde da Família: estudo sobre o processo de implementação em município do Rio de Janeiro a partir do modelo ambigüidade-conflito. 2006. 129 f. Tese (Doutorado em Saúde Pública) - Fundação Oswaldo Cruz, Escola Nacional de Saúde Pública Professor Sérgio Arouca, Rio de Janeiro, 2006.

\section{CONSELHO NACIONAL DE SECRETÁRIOS DE}

SAÚDE. Assistência Farmacêutica no SUS. Brasília, DF:

CONASS, 2011. 186 p.

\section{INSTITUTO BRASILEIRO DE GEOGRAFIA E}

ESTATÍSTICA (IBGE). Conta-satélite de saúde do Brasil: 2005-2007. 2009. Disponível em: <http://www. ibge.gov.br/home/estatistica/economia/economia_saude/css_2005_2007/economia_saude.pdf>. Acesso em: 22 mar. 2015.

. Estatísticas do Cadastro Central de Empresas: 2008/IBGE - Gerência do Cadastro Central de Empresas. Rio de Janeiro, RJ: IBGE, 2010. 167 p. Disponível em: $<$ http://biblioteca.ibge.gov.br/visualizacao/livros/liv44967.pdf >. Acesso em: 5 abr. 2015.

LE GRAND, A.; HOGERZEIL, H. V.; HAAIJERRUSKAMP, F. M. Intervention in rational use of drugs: a review. Health policy and planning, New York, v. 14, n. 2, p. 89-102, 1999.

MARIN, N. et al. Assistência farmacêutica para gerentes municipais. Rio de Janeiro: Opas/OMS, 2003.

MATLAND, R. E. Synthesizing the implementation literature: the ambiguity-conflict model of policy implementation. Journal of Public Administration Research and Theory, Lawrence, v. 5, n. 2, p. 145-174, abr. 1995.

NASCIMENTO, M. C. Medicamentos: ameaça ou apoio à saúde? Vantagens e perigos do uso de produtos da indústria farmacêutica mais consumidos no Brasil: vitaminas, analgésicos, antibióticos e psicotrópicos. Rio de Janeiro: Vieira \& Lent, 2003.

\section{ORGANIZAÇÃO PAN-AMERICANA DA SAÚDE} (OPAS); ORGANIZAÇÃO MUNDIAL DA SAÚDE. Avaliação da assistência farmacêutica no Brasil. Brasília, DF: Organização Pan-Americana da Saúde, 2005.

\section{SABATIER P. A.; JENKINS-SMITH H. C. Policy} change and learning. An advocacy coalition approach. Colorado: Ed. Westview Press, 1993.

TONG, A.; SAINSBURY, P.; CRAIG, J. Consolidated criteria for reporting qualitative research (COREQ): a 32 -item checklist for interviews and focus groups. Int $J$ Qual Health Care, New York, v. 19, n. 6, p. 349-357, 2007.

SANTOS, V.; NITRINI, S. M. O. O. Indicadores do uso de medicamentos prescritos e de assistência ao paciente de serviços de saúde. Revista de Saúde Pública, São Paulo, v. 38, n. 6, p. 819-834, dez. 2004.

VIEIRA, F. S.; ZUCCHI, P. Financiamento da Assistência Farmacêutica no Sistema Único de Saúde. Revista Saúde Soc., São Paulo, v. 22, n. 1, p. 73-84, 2013.

\section{WORLD HEALTH ORGANIZATION (WHO). WHO} medicines strategy: countries at the core - 2004-2007. Geneva: WHO Press, 2004.

\section{WORLD HEALTH ORGANIZATION (WHO).}

Promoting rational use of medicines: core components. (WHO Policy Perspectives on Medicines No. 5). Geneva, 2002. Disponível em: <http://apps. who.int/iris/bitstream/10665/67438/1/WHO_ EDM_2002.3.pdf $>$. Acesso em: 24 abr. 2015.

\footnotetext{
Recebido para publicação em abril de 2015

Versão final em setembro de 2015

Conflito de interesses: inexistente

Suporte financeiro: não houve
} 